\title{
Increasing Distributed Generation Penetration using Soft Normally-Open Points
}

\author{
Jeffrey M. Bloemink, Student Member, IEEE and Timothy C. Green, Senior Member, IEEE
}

\begin{abstract}
This paper considers the effects of various voltage control solutions on facilitating an increase in allowable levels of distributed generation installation before voltage violations occur. In particular, the voltage control solution that is focused on is the implementation of 'soft' normally-open points (SNOPs), a term which refers to power electronic devices installed in place of a normally-open point in a medium-voltage distribution network which allows for control of real and reactive power flows between each end point of its installation sites. While other benefits of SNOP installation are discussed, the intent of this paper is to determine whether SNOPs are a viable alternative to other voltage control strategies for this particular application. As such, the SNOPs ability to affect the voltage profile along feeders within a distribution system is focused on with other voltage control options used for comparative purposes. Results from studies on multiple network models with varying topologies are presented and a case study which considers economic benefits of increasing feasible DG penetration is also given.
\end{abstract}

Index Terms-distributed generation, DG, DG capacity, DG penetration, embedded generation, soft normally open points, SNOP

\section{INTRODUCTION}

$\mathbf{T}$ HE integration of distributed generators (DG), such as wind turbines, co-generation and photovoltaics poses a number of problems, increasingly so as the total capacity installed in a particular network rises [1]. The issues of note consist of voltage violations, loss impacts, power quality effects, protection considerations, and reliability concerns [1]. The key limiting factor in large scale DG integration, however, remains the voltage-rise effect on network buses [2]. In order to minimize the overall effect on voltage, network operators prefer to connect DGs at higher voltages where their impact on voltage levels is negligible; but as connection costs increase considerably at higher voltages, developers tend to prefer to connect their equipment at lower voltages, i.e. the $11 \mathrm{kV}$ or medium-voltage distribution system [3] where the effects of DG installation on network voltages are particularly prevalent [4]. It is for this reason that the study presented in this paper considers the issue of voltage violations due to increasing DG penetration and how different voltage control strategies perform in its mitigation.

For most network configurations, the worst case scenario for the voltage profile issue will be that in which the distribution system in question is operating at minimum load with DGs at

This work is supported by the FLEXnet project, part of the Engineering and Physical Sciences Research Council (EPSRC) Supergen Consortium.

J. Bloemink and T. Green are with the Electrical Engineering dept. at Imperial College, London, UK (emails: jeffrey.bloemink08@imperial.ac.uk, t.green@imperial.ac.uk) maximum output [3], [5], [4]. Usually, increasing DG capacity will eventually cause a violation of upper voltage limits; however, when compensatory devices have been implemented in the network (such as on-load tap changers (OLTC), loadratio control transformers, or other devices which attempt to regulate system voltage levels based on load) other undesirable effects could be seen, i.e. lower limit violations on other feeders without any DG installed [6].

This paper considers multiple voltage control strategies but is primarily focused on assessing the relative benefit for increasing DG penetration in the presence of 'soft' normallyopen points (SNOPs). The intention is to establish whether SNOPs are a viable solution to the voltage violation issues raised by large scale DG integration. The term SNOP refers to a device installed in place of a normally-open (NOP) or normally-closed point (NCP) that can control real and reactive power flow through each of its end-nodes. This is illustrated in Fig. 1. A SNOP could be implemented with back-to-back (ACAC) voltage-sourced converters (VSCs) synchronized with the AC network.

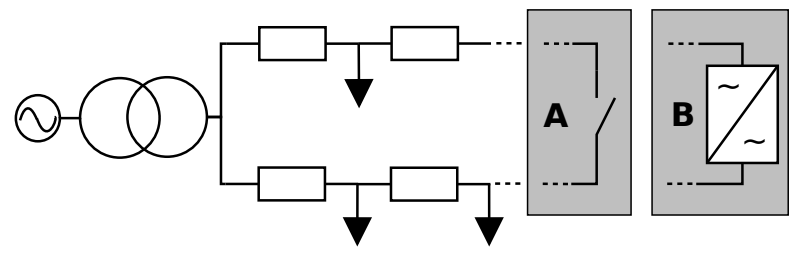

Figure 1. Single line diagram of a simple distribution system option A representing a NOP connection and $\mathrm{B}$ representing a SNOP

The intention of a SNOPs is to provide a significant increase in the flexibility of current distribution networks with an insignificant level of equipment or infrastructure upgrades. Distribution systems are typically classified as radial or meshed systems, each with their own inherent advantages and disadvantages [7]. By placing SNOPs into a distribution system, a hybrid system type is formed possessing the benefits of both radial and meshed systems (simplified protection scheme and high reliability) with the primary drawbacks (lack of isolation between feeders) removed. Other key features and properties of a distribution system containing SNOPs are:

- support to loads isolated due to a fault on a feeder can be provided immediately through power transfer from another feeder connected via a SNOP (as opposed to a NOP which takes time to close)

- voltage at SNOP interface terminals can be regulated to a certain level or reactive power set points can be assigned 
as required

- real power flow between feeder lines can be controlled for improved feeder load balancing and loss reductions

- disturbances and faults on one feeder can be isolated from other feeders connected to it via SNOPs

- with voltage-sourced converter over current limiting, contributions to fault currents are small (and controllable, if necessary for the protection system to operate correctly)

- voltages throughout the system can be controlled by changing the real and reactive power flow through the SNOP, allowing for increased levels of DG integration

The voltage control capability is the feature being considered in this paper. By being able to affect the voltage profile throughout the distribution system, SNOPs can be operated with the objective loss minimization while still constraining the voltage to pre-specified limits.

\section{Procedure And Modeling}

\section{A. Distributed Generation Levels and Placement}

For the purposes of this report, DG penetration results are given as a percentage of the maximum loading of the system under study. For example, in a distribution system operating at $25 \%$ of peak load with a DG penetration of $100 \%, 75 \%$ of that power will be exported back to the transmission system. If a system is operating at peak load with a DG penetration of $25 \%, 75 \%$ of the power will be coming from the transmission system rather than from the locally installed generation.

As previously mentioned, the worst case scenario for DG penetration is widely recognized as when the network is operating with minimum load and maximum (full) DG output [3], [5], [4]. In this study, maximum allowable DG penetration is calculated at this worst case scenario. The minimum load for each data set was calculated using typical yearly load profile data, obtained from [8], for a particular customer type and applied using approximations to establish the dominant type of customer at each bus.

Rather than considering specific scenarios for the placement of DG throughout the networks under study, DG placement over time was considered to be a random process. Scenarios in which small quantities of generation are dispersed randomly throughout the network were considered, as well as the clustering of generation into certain network nodes. The two placement strategies are illustrated in Fig. 2. The random dispersal scenario is intended to represent placement evolution where many small generators with many owners are placed throughout the system, whereas the intention of the clustered placement strategy is to represent large DG installations with single owners or operators. For brevity, results reported in this paper are for clustered placement near to feeder endpoints as large concentrations of DG were observed to cause voltage violations at lower penetration levels, and so they can be considered as the more challenging scenario. When relevant, the results for random dispersal will be discussed.

To account for many possible network configurations as DG installations occur over time, several iterations of the study are performed in which DG is dispersed randomly and incrementally in varying quantities. These random processes

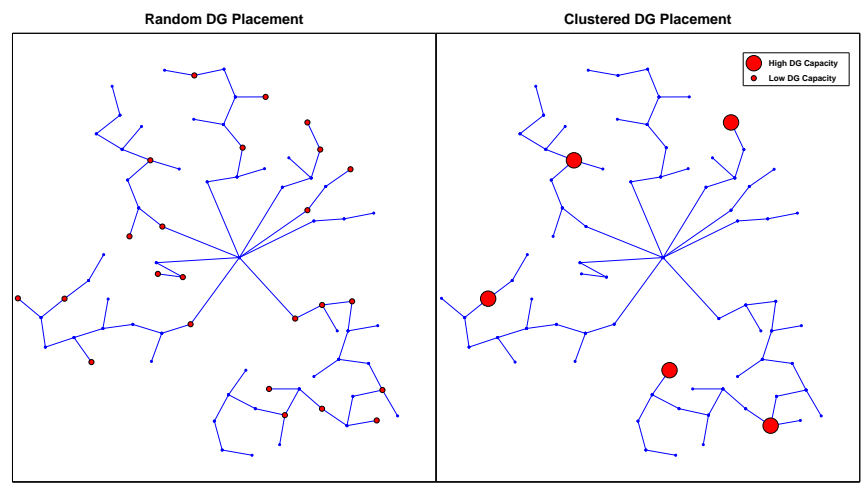

Figure 2. Graphical representation of different DG placement evolutions considered on a given distribution system (urban)

cause results to vary between each iteration, as illustrated in Fig. 3 where the statistical distribution of the maximum DG penetration results is shown. Results quoted in this paper are the mean maximum DG penetration.

Clustered dispersal tends to result in a lower standard deviation than random dispersal due to the decreased number of buses considered for DG placement, and hence a reduction in possible eventual configurations for DG installation. The reader should consider that there would likely be some degree of planning when installing DG, rather than allowing completely arbitrary placement, hence configurations that cause voltage violation problems at lower DG penetration levels would be eliminated. Accounting for planning, the standard deviation would further decrease, and the mean would shift to the right due to removal of possible configurations with unacceptably low maximum DG penetration levels. The effect of proper planning is beyond the scope of this paper, but its effect should be considered when interpreting Fig. 3.
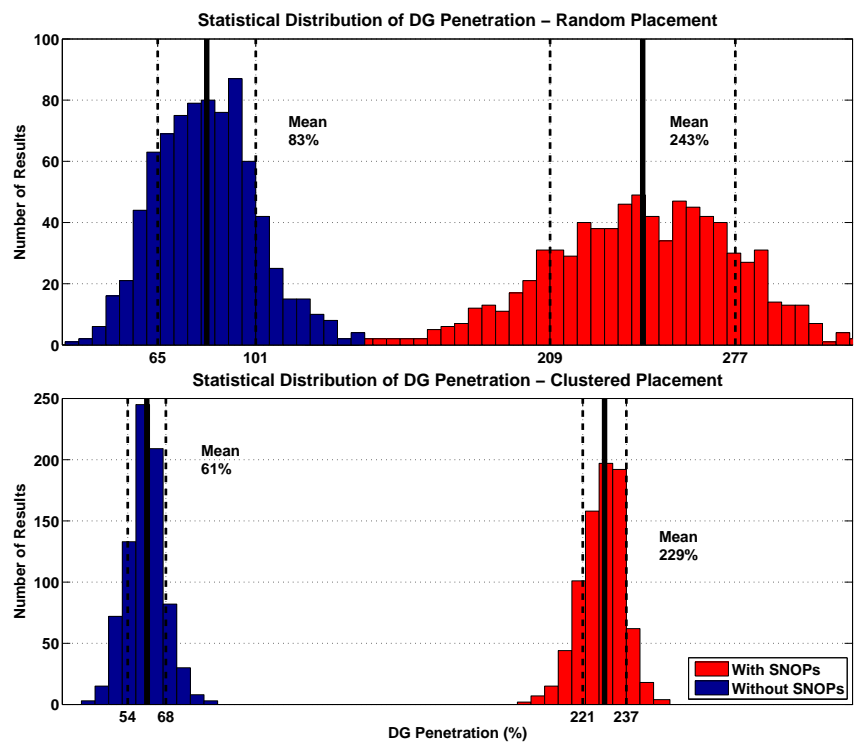

Figure 3. Statistical distribution for urban distribution system with and without SNOPs installed based on 800 iterations of the study 


\section{B. SNOP Placement and Rating}

A previous study was performed that involved determining the best location for SNOPs, based on a given number of possible candidate installation sites, with a loss minimization objective in mind,. Feeder branch ends were chosen as candidate sites as they maximize SNOP emergency benefits for a radially connected system because the number of loads supplied through SNOP for a fault isolated nearest to the bulksupply point is greatest. Regarding the number of SNOPs installed: previous results indicated marginal reductions in losses for three SNOP units installed versus two in all systems considered, so two SNOPs units are used in this paper.

The hypothesis was made that SNOP placement which yielded greatest control over losses would also yield greatest control over the overall voltage profile of the system, thereby increasing the ability to accommodate greater DG capacity. To test this, different SNOP placements were considered in simulation and results were found to agree with the hypothesis; that is, sites that yielded the worst results in terms of loss minimization also yielded poorer results for controlling voltage profiles and, in turn, resulted in lower feasible DG penetration levels.

The SNOP rating required to achieved certain objectives must also be considered as it will affect the total cost of installation, with the price of a SNOP unit increasing with the power rating; however, maximizing DG penetration will not likely determine the required maximum rating for the SNOP units in most cases. The maximum rating will likely be determined by the emergency power transfer capability required, i.e. when it must supply power to the loads on an adjacent feeder due to isolation of a fault. SNOP ratings of 5 MVA and 10 MVA were considered in the studies presented in this paper. These ratings are intended to be on the order of the emergency power transfer capability required to support SNOP-connected feeders. They are also on the order of AC-AC conversion systems used in traction and wind power applications at medium-voltage levels, and therefore considered feasible.

\section{Networks Under Study}

A total of 10 different distribution networks were studied, using generic data from the UKGDS (United Kingdom Generic Distribution System) project [8] and real data from EDF Energy Networks Ltd., a distribution network operator (DNO) operating in the UK. The generic systems are classified according to their intended system type, that is: rural, urban and mixed. The real systems, using data from EDF are classified separately as real but tend to exhibit characteristics of combined or urban networks, i.e. high load density. They have been classified separately to emphasize the fact that the data sets and simulation results have been obtained for existing distribution networks.

- Rural systems are characterized by long feeders with relatively light loading per bus ( $25 \mathrm{~kW} / \mathrm{bus}$ average). Two data sets were studied representing rural system types, designated Rural 1 and Rural 2 within this report
- Urban distribution systems are characterized by their high loading density and large total load. Only one generic data set representing this system type was available, designated Urban 1; however the real distribution system data shares similar characteristics, as mentioned

- A mixed distribution contains combined characteristics of rural and urban system types, with some rural areas and some urban areas. Four data sets were used representing this system type, designated Mixed 1, Mixed 2, Mixed 3 and Mixed 4

- The real data sets from three different areas are designated as Real 1, Real 2 and Real 3

\section{Network Model}

A general formulation used for representing the distribution systems under study was developed and used. This formulation allows for explicit calculation of node voltages, load currents, as well as the branch voltages used in network loss calculation. Formulating the model this way makes it possible to perform quick, procedural, modifications to network topology and various other aspects in order to determine their effect on system operation, i.e. modification of loading, addition of generators, adding branches for meshing.

The variables in the model are the branch currents, $\vec{i}$, the load currents, $\vec{i}_{l}$, the node voltages, $\vec{v}$, and the substation (or slack bus) voltage, $v_{s}$. A vector, $\vec{x}$, contains decision variables for the optimisation procedure such as direct- and quadratureaxis currents injected by the power converters of the SNOPs. The variables are related by the electrical network equations which are briefly summarised here in which the electrical parameters and branch connections are combined in matrices $\mathbf{U}, \mathbf{K}, \mathbf{J}, \mathbf{A}, \mathbf{S}$, and $\mathbf{L}$. While vector and matrix elements are presented as complex, it is possible to formulate using real numbers by simple extension.

The network currents and voltages are described by the following,

$$
\begin{aligned}
0 & =\mathbf{U} \vec{i}+\mathbf{K} \overrightarrow{i_{l}}+\mathbf{J} \vec{x} \\
\vec{i} & =\mathbf{A} \vec{v}+\mathbf{S} v_{s} \\
\vec{i}_{l} & =\mathbf{L} \vec{v} .
\end{aligned}
$$

Setting

$$
\begin{aligned}
\mathbf{O} & =\left(-\mathbf{A}(\mathbf{U A}+\mathbf{K L})^{-1} \mathbf{U S}+\mathbf{S}\right) \\
\mathbf{P} & =-\mathbf{A}(\mathbf{U A}+\mathbf{K L})^{-1} \mathbf{J} \\
\mathbf{M} & =-(\mathbf{U A}+\mathbf{K L})^{-1} \mathbf{U S} \\
\mathbf{N} & =-(\mathbf{U A}+\mathbf{K L})^{-1} \mathbf{J}
\end{aligned}
$$

we have, after simplification, the voltage and current expressed in terms of the slack bus voltage and the soft normallyopen point current,

$$
\begin{aligned}
\vec{v} & =\mathbf{M} v_{s}+\mathbf{N} \vec{x} \\
\vec{i} & =\mathbf{O} v_{s}+\mathbf{P} \vec{x} .
\end{aligned}
$$




\section{E. Optimization Objective Function}

Studies on networks with objective functions considered for the installed SNOPs have indicated success in meeting an optimization objective, such as loss minimization or load balancing, in addition to satisfying the required constraints with rising DG penetration. Some results from these studies are shown in Fig. 4. The figure illustrates how the voltage profile is affected by the optimization objective being used. e.g the objective function for loss minimization is

$$
f(x)=\vec{i}^{H} \mathbf{R} \vec{i},
$$

where $\mathbf{R}$ is a matrix with containing the resistance of the relevant branch and ${ }^{H}$ denotes the complex transpose. This formula can be rewritten in terms of the optimization variable. Substituting from (5), a quadratic objective function is formed:

$$
f(x)=\vec{x}^{H} \mathbf{P}^{H} \mathbf{R} \mathbf{P} \vec{x}+2 v_{s} \mathfrak{R e}\left\{\mathbf{O}^{T} \mathbf{R} \mathbf{P} \vec{x}\right\}+\mathbf{O}^{H} \mathbf{R O}\left|v_{s}\right|^{2} .
$$

Note that the constraints discussed in Section II-F are all non-linear, and hence a sequential quadratic programming (SQP) method is necessary for solution of the optimization problem [9].

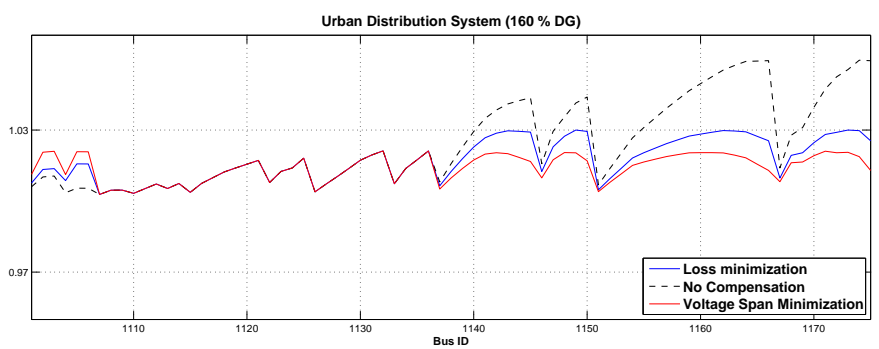

Figure 4. Urban distribution system voltage profile with different optimizer objectives

It should be noted that. for the purposes of the studies considered in this paper, an optimization objective is not utilized. This was done in order to improve convergence time due to the slackened solution requirements, i.e. merely satisfying constraints rather than a measure of optimality. This omission of functionality was deemed reasonable for a study on increasing DG penetration because, in practice, secondary objectives for the SNOPs such as loss minimization would be abandoned in favour of more important functions, such as eliminating voltage violations.

\section{F. System Constraints}

The first constraint considered is the real power balance between two ends of the SNOP, i.e. power in should be equal to power out plus losses for each SNOP. The power balance constraints can be expressed as follows:

$$
\vec{v}^{T} \mathbf{Y} \vec{x}=0
$$

Where $\mathbf{Y}$ is a matrix constructed to form a power balance equation for soft normally-open points of the system, such that the sum of all real power in SNOP pairs is zero.
Network voltages are limited to within 0.03 per-unit of nominal according to:

$$
\vec{V}_{\text {min }} \leq \vec{v} \leq \vec{V}_{\max }
$$

The voltage constraint is intended to keep bus voltages throughout the network within pre-specified limits (between 0.97 and 1.03 p.u. was considered for all systems). Satisfying these constraints counters the voltage-rise problem associated with DG installation.

A constraint on the SNOP output voltage is also added to account for device limitations, according to:

$$
\vec{v}^{T} \mathbf{Y} \leq \vec{V}_{S N O P, \max }
$$

The SNOP rating is limited according to:

$$
\left\|\vec{S}_{S N O P}\right\| \leq \vec{S}_{S N O P, \max }
$$

where $S_{S N O P}$ is a vector representing the apparent power of each end of the SNOP units and $\vec{S}_{S N O P, \max }$ is set according to the study. In this paper, both 5 MVA and 10 MVA SNOP units are considered.

Regarding current constraints: it is assumed that feeders will be upgraded or split and protection equipment upgraded as necessary with increasing DG penetration levels, so current level constraints were not considered for the results presented in this paper. Current constraints, or rather, possible upgrades, will depend on the system under study and should be considered in more detail when finalizing feasible DG penetration results for a particular system. It should be noted that, with line upgrades, the resistive effect of the feeder will be diminished, i.e. line resistance per unit length decreases with increasing ampacity of the cable. If this were modeled, the reduction in line resistance would result in further increased penetration levels due to a reduction in voltage drops and rises along feeders. Studies presented in this paper have not modeled this 'line upgrade effect', as it represents a worst-case scenario.

\section{G. Load and Distributed Generator Modeling}

For the studies presented in this report, DG units were modeled as constant-MVA sources. The power factor for each DG unit was set randomly between 0.9 leading and 0.9 lagging to represent the present most likely case [10]. Further detail in DG modeling was omitted to reduce the complexity of the simulations. It is assumed that the DG-units are passive sources, in the sense that they do not coordinate with loading of the distribution system or perform any kind of voltage regulation themselves. As such, their injection of real and reactive power into the network could lead to undesired effects on the voltage profile of the line which can only be mitigated by planning on a 'worst case' basis.

Loads are represented partly as linear, or constantimpedance, and partly as non-linear, or constant-MVA, elements according to their status as predominantly commercial/residential loads or industrial/agricultural loads [11].

For solving the load-flow problem, load-flow technique was developed which takes advantage of the network model formulation, allowing for representation of loads as partially 
constant-impedance and partially constant-MVA in order to more accurately represent their non-linear behaviour. This method varies from commonly used load-flow techniques, (e.g. Gauss-Seidel, Newton-Raphson) in the sense that it varies load impedance according to error in bus power calculations rather than varying voltages.

\section{H. Voltage Control Strategies}

In present UK distribution networks, compensation for voltage drop due to loading is often achieved by an Onload Tap Changing (OLTC) device at the bulk-supply point, sometimes employing state estimation or data acquisition from measurements at line end-points in order to determine whether a tap operation is necessary [4].

A typical strategy for determining set points is the following: when at minimum load, the tap changer is adjusted to the maximum level possible before violating upper voltage limits, i.e. as close as possible to the set point designated for maximum loading. This mininizes the number of taps traversed to compensate for line voltage drops when the network reaches maximum loading; in turn, reducing the change in voltage experienced by the network for abrupt loading changes and wear on OLTC devices [12]. In this paper, the typical strategy is modified to cope with increasing DG penetration rather than assume that the OLTC set points would not be adjusted as DG is installed over time. The OLTC is otherwise operated as in [12], however the tap set point at minimum load is permitted to lower to a minimum level defined by the average network loading, resulting in a greater range of allowed operation. This is a compromise between the reduction of OLTC variation and the accommodation of DG, i.e. the greater range allows for more DG capacity while limits imposed still attempt to reduce tap and voltage changes. These allowed minimum set points are precalculated for each network under study. The tap changer modeled is capable of varying between 0.9 and 1.1 p.u. with a resolution of 30 steps. The compromised strategy and OLTC model was used for all cases other than the dynamic OLTC to be described.

The other strategies being compared with SNOPs consist of solutions that don't require mass coordination or communication between devices, i.e. reactive power compensation at the DG site will compensate for local reactive power flows caused by DG, but won't coordinate with other compensation devices to optimize voltage levels. SNOP units coordinate with each other to meet the network constraints, and also with the OLTC in the dynamic OLTC control scenario, but this is not considered as mass coordination.

1) Passive Network: The OLTC present in the distribution network is operated as described above. So, the tap changer output voltage a minimum loading is set to minimize the difference between the maximum loading set point and still accommodate increasing DG by allowing adjustments as low as the average loading set point.

2) Dynamic OLTC: The OLTC is modified or replaced with a conceptual dynamic OLTC device to allow for relatively quick response to requirements for voltage compensation. It is assumed that there is no penalty for changing taps, or the penalty is not considered due to device improvements or otherwise. It is referred to as dynamic since it is intended to be able to change its tap setting according to DG output levels and loading condition as needed throughout the day. This effect is achieved by calculating the tap changer output voltage required to keep voltage within the designated limits as DG penetration increases. The effect of the dynamic OLTC will be considered on its own as well as in coordination with installed SNOPs. The Dynamic OLTC is also considered to have an increased tap resolution (100) in order to achieve a more continuous output, and to facilitate coordination with the SNOPs in the optimization algorithm, as in [9].

3) Reactive Power Compensation at SNOP site : In this scenario, the presence of some form of reactive power compensation such as an SVC or STATCOM, with the same apparent power capability, i.e. total MVA, is used in place of a SNOP. Results are intended to be an indicator as to whether DG penetration increases provided by the presence of SNOPs is merely due to its reactive power compensation capability or if the routing of real power provides any added benefit. The uncompensated columns of Table III for this scenario will be identical to that of Passive Network case as it is effectively the same situation.

4) Meshed at SNOP Site: To model this scenario, feeder branches (representing normally-closed points) are inserted between several nodes throughout the system. Normally-closed points were selected based on the previously determined candidate sites for SNOP installation, which tended to be towards feeder endpoints. Note that this scenario involves increasing the degree of meshing considerably where all primary feeders have a normally-closed point to one-another.

In addition to meshing, reactive power compensation devices are also inserted at the same SNOP sites as in the strategy described in Section II-H3, and their effect is considered.

5) Unity Power Factor DGs: A possibility for the increase in maximum DG penetration is to have the DNO install, or require installation of some kind of reactive power compensation at the DG site itself or to have each DG interfaced with a unity power factor correction interface. To achieve this in simulation reactive power output for DGs is set to zero.

\section{Results AND Discussion}

This section presents and discusses the results of the various studies that have been performed in order to demonstrate how the presence of SNOPs affects the DG penetration achievable for a given distribution network, and how SNOPs compare with other solutions. All simulation results discussed in this section are summarized in Table III. The uncompensated column indicates scenarios in which no power electronic compensation devices (SNOPs, SVCs) are operating, while the compensated column contains results for networks with compensation devices operating with the designated rating. Note that the reactive power compensation at SNOP site is referred to as the $S V C$ case in the table.

As evident from Table III, increasing the rating of the installed SNOPs from 5 MVA to 10 MVA results in higher achievable DG penetration levels. It can also be seen amongst 
several systems studied that with higher SNOP rating, the effect of combined strategies, such as utilizing the dynamic OLTC with SNOPs, provides only marginal benefits over merely using the SNOPs with the passive network case. With lower SNOP rating, the added benefit of combining voltage control strategies is more evident.

\begin{tabular}{|c|c|c|c|c|}
\hline & & Uncompensated & Comp & sated \\
\hline Data Set & Control & - & 5 MVA & 10 MVA \\
\hline \multirow{5}{*}{$\begin{array}{c}\text { Real 1 } \\
(23.7 \mathrm{MW})\end{array}$} & Passive & $8.9 \%$ & $140.0 \%$ & $217.9 \%$ \\
\hline & $S V C$ & - & $65.5 \%$ & $67.1 \%$ \\
\hline & Unity $P F D G$ & $10.9 \%$ & $152.3 \%$ & $210.6 \%$ \\
\hline & Dyn. OLTC & $35.3 \%$ & $140.3 \%$ & $250.3 \%$ \\
\hline & Meshed & $28.5 \%$ & $423.4 \%$ & $423.8 \%$ \\
\hline \multirow{5}{*}{$\begin{array}{c}\text { Real 2 } \\
(22.7 \mathrm{MW})\end{array}$} & Passive & $12.4 \%$ & $105.4 \%$ & $133.3 \%$ \\
\hline & $S V C$ & - & $50.9 \%$ & $58.5 \%$ \\
\hline & Unity $P F D G$ & $14.6 \%$ & $110.0 \%$ & $137.5 \%$ \\
\hline & Dyn. OLTC & $33.7 \%$ & $105.3 \%$ & $136.6 \%$ \\
\hline & Meshed & $52.7 \%$ & $236.7 \%$ & $236.8 \%$ \\
\hline \multirow{5}{*}{$\begin{array}{c}\text { Real 3 } \\
(37.5 \mathrm{MW})\end{array}$} & Passive & $27.4 \%$ & $119.7 \%$ & $159.9 \%$ \\
\hline & $S V C$ & - & $94.4 \%$ & $109.2 \%$ \\
\hline & Unity $P F D G$ & $37.5 \%$ & $125.7 \%$ & $164.2 \%$ \\
\hline & Dyn. OLTC & $78.6 \%$ & $119.5 \%$ & $161.2 \%$ \\
\hline & Meshed & $93.6 \%$ & $508.7 \%$ & $514.6 \%$ \\
\hline \multirow{5}{*}{$\begin{array}{c}\text { Rural 1 } \\
(9.13 \mathrm{MW})\end{array}$} & Passive & $87.0 \%$ & $311.5 \%$ & $537.1 \%$ \\
\hline & $S V C$ & $87.0 \%$ & $262.8 \%$ & $500.6 \%$ \\
\hline & Unity $P F D G$ & $102.2 \%$ & $361.8 \%$ & $579.4 \%$ \\
\hline & Dyn. OLTC & $167.1 \%$ & $360.7 \%$ & $537.0 \%$ \\
\hline & Meshed & $100.4 \%$ & $302.2 \%$ & $611.8 \%$ \\
\hline \multirow{5}{*}{$\begin{array}{c}\text { Rural 2 } \\
(8.30 \mathrm{MW})\end{array}$} & Passive & $95.7 \%$ & $344.1 \%$ & $570.3 \%$ \\
\hline & $S V C$ & - & $279.1 \%$ & $551.8 \%$ \\
\hline & Unity $P F D G$ & $104.5 \%$ & $401.1 \%$ & $673.4 \%$ \\
\hline & Dyn. OLTC & $166.8 \%$ & $416.4 \%$ & $672.2 \%$ \\
\hline & Meshed & $123.8 \%$ & $361.2 \%$ & $715.1 \%$ \\
\hline \multirow{5}{*}{$\begin{array}{c}\text { Urban 1 } \\
(24.3 \mathrm{MW})\end{array}$} & Passive & $74.5 \%$ & $210.3 \%$ & $376.4 \%$ \\
\hline & $S V C$ & - & $205.6 \%$ & $360.3 \%$ \\
\hline & Unity $P F D G$ & $88.5 \%$ & $259.8 \%$ & $388.4 \%$ \\
\hline & Dyn. OLTC & $130.5 \%$ & $276.0 \%$ & $382.7 \%$ \\
\hline & Meshed & $113.5 \%$ & $228.7 \%$ & $384.1 \%$ \\
\hline \multirow{5}{*}{$\begin{array}{c}\text { Mixed 1 } \\
(16.8 \mathrm{MW})\end{array}$} & Passive & $80.9 \%$ & $249.4 \%$ & $433.6 \%$ \\
\hline & $S V C$ & - & $191.7 \%$ & $351.5 \%$ \\
\hline & Unity $P F D G$ & $90.8 \%$ & $281.7 \%$ & $512.3 \%$ \\
\hline & Dyn. OLTC & $136.3 \%$ & $312.0 \%$ & $500.2 \%$ \\
\hline & Meshed & $150.1 \%$ & $299.0 \%$ & $499.3 \%$ \\
\hline \multirow{5}{*}{$\begin{array}{c}\text { Mixed 2 } \\
(13.9 \mathrm{MW})\end{array}$} & Passive & $60.0 \%$ & $146.8 \%$ & $229.1 \%$ \\
\hline & $S V C$ & - & $122.0 \%$ & $213.5 \%$ \\
\hline & Unity $P F D G$ & $67.6 \%$ & $166.1 \%$ & $261.7 \%$ \\
\hline & Dyn. OLTC & $92.9 \%$ & $193.7 \%$ & $295.5 \%$ \\
\hline & Meshed & $174.8 \%$ & $223.7 \%$ & $287.3 \%$ \\
\hline \multirow{5}{*}{$\begin{array}{c}\text { Mixed 3 } \\
(19.7 \text { MW) }\end{array}$} & Passive & $38.0 \%$ & $147.3 \%$ & $252.9 \%$ \\
\hline & $S V C$ & - & $130.6 \%$ & $239.9 \%$ \\
\hline & Unity $P F D G$ & $44.6 \%$ & $175.0 \%$ & $277.6 \%$ \\
\hline & Dyn. OLTC & $61.0 \%$ & $190.6 \%$ & $274.6 \%$ \\
\hline & Meshed & $59.5 \%$ & $139.6 \%$ & $255.4 \%$ \\
\hline \multirow{5}{*}{$\begin{array}{c}\text { Mixed 4 } \\
(19.3 \mathrm{MW})\end{array}$} & Passive & $38.3 \%$ & $123.0 \%$ & $241.3 \%$ \\
\hline & $S V C$ & - & $114.3 \%$ & $228.1 \%$ \\
\hline & Unity $P F D G$ & $42.2 \%$ & $141.4 \%$ & $290.8 \%$ \\
\hline & Dyn. OLTC & $71.3 \%$ & $169.8 \%$ & $287.7 \%$ \\
\hline & Meshed & $52.4 \%$ & $129.5 \%$ & $239.0 \%$ \\
\hline
\end{tabular}

Table I

ACHIEVABLE DG PENETRATION LEVELS FOR VOLTAGE CONTROL SCENARIOS CONSIDERED

\section{A. Voltage Control Strategy Comparison}

The cases discussed are as follows:

- Passive network without SNOPs (reference case)
- Network with additional normally closed points/meshing near to feeder ends

- Reactive power compensation at DG site without SNOPs (Unity power factor DG)

- Reactive power compensation only at SNOP site (SVC at SNOP site)

- Dynamic OLTC without SNOPs present

- Passive network compensated with SNOPs

- Dynamic OLTC with SNOPs present

While other combinations of strategies exist and are presented in Table III, they are not discussed as they are considered unlikely due to either marginal improvements or extensive network upgrades required.

The results of the listed scenarios for clustered DG placement and 5 MVA SNOPs are shown graphically in Fig. 5. The scenario combining SNOPs with the Dynamic OLTC allows for the greatest DG penetration, followed by SNOPs with a passive network, reactive power compensation at SNOP site strategy, dynamic OLTC strategy, heavy meshing, and finally the unity power factor compensation at each DG site. A similar trend is observed for the random DG placement scheme.

In many cases, the dynamic OLTC combined with the SNOP yields the best results, however they appear to be incremental compared to the passive network compensated only with SNOPs. Cost-benefit analysis for the particular case would be necessary to determine if the incremental benefit provided by having both devices is necessary and feasible. There are also several cases in which heavy meshing with reactive power compensation devices provides the best results, but the disadvantages to heavily meshed networks should be considered when evaluating this option. This table also considers other combinations of voltage control strategies, i.e. reactive power compensation at DG site with SNOPs.

\section{B. Distributed Generator Placement}

Though the results are not shown in this paper, it was found that random DG dispersal results in the highest achievable DG penetration for all data sets considered for the voltage control scenarios of interest. This is intuitive, as the voltage rise effect from DG installation is spread throughout the network buses in small quantities rather than localized at fixed DG installation points.

For the study of clustered DG placement, maximum DG penetration in compensated systems tends to be higher for clustered placement strategy where clusters are located close to SNOPs, as opposed to placing the clusters arbitrarily throughout the networks. It is likely that the sites chosen to install such large clusters of generation would be done so with more scrutiny, so it is possible that greater penetration could be achieved with placement considerations made by the DG developer or the DNO.

\section{Variation Amongst System Types}

The system types considered contain different characteristics and thus DG installation will affect the systems differently. For every voltage control strategy and system type considered, Table III indicates considerable increase in maximum DG 


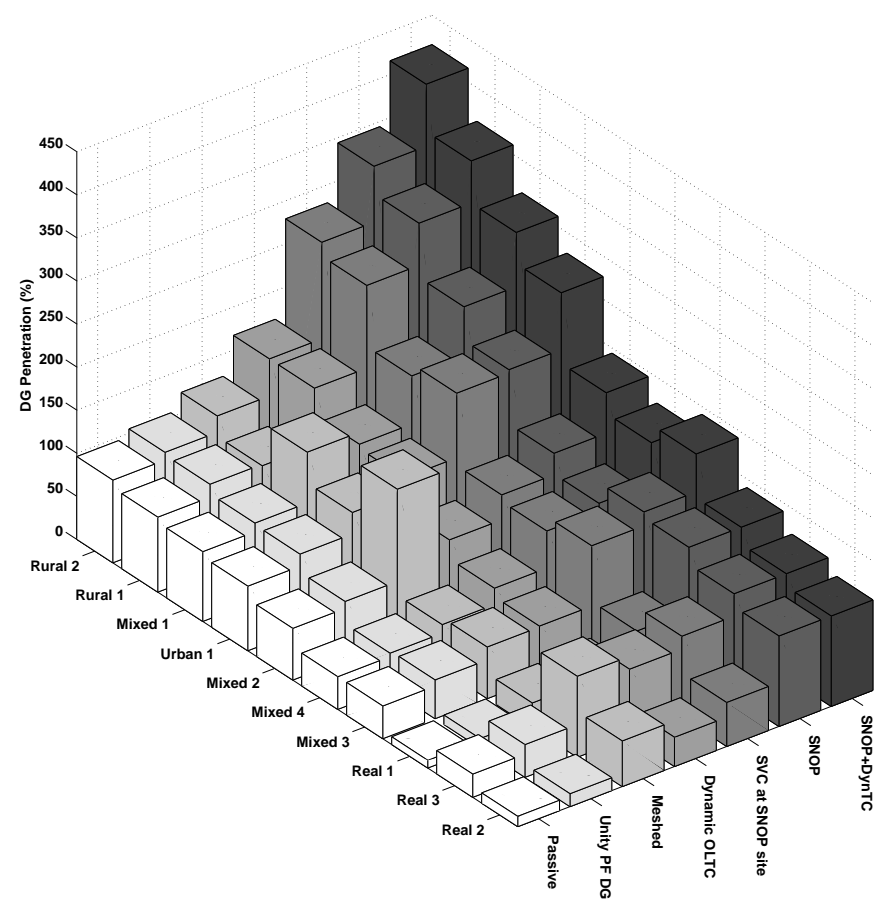

Figure 5. Graphical representation of DG penetration results with different voltage control strategies for 5 MVA SNOPs with DG placement clustered at feeder endpoints

penetration when compared with reference case. Recall that DG penetration figures are quoted in percentages of maximum loading and rural systems tend to be more lightly loaded, so $200 \%$ penetration for a rural system would translate to a lower capacity than $100 \%$ penetration for an urban system type. For example, data set Rural 1 is able to achieve $311 \%$ capacity with 5 MVA SNOPs installed, and Urban 1 is able to achieve $210 \%$. This translates to approximately $28 \mathrm{MW}$ and $52 \mathrm{MW}$ installed capacity, respectively; noting that the urban system has higher capacity with a lower percentage.

Rural distribution systems tend to have lower geographical load density and hence will be open to more installed DG capacity. So, being able to increase DG capacity in rural systems well beyond $100 \%$ is critical for accommodating distributed generation. Utilizing SNOPs with higher power ratings in conjunction with dynamic OLTCs allows penetration to be further increased to over $500 \%$ for Rural 1, so for rural networks, combinations of voltage control strategies may be necessary to achieve desired DG penetration levels.

Maximum DG penetration results shown here are consider the worst case scenario (minimum loading, maximum DG output condition); however, the results do not consider the intermittent nature of the installed DG (i.e. wind) or the likelihood of maximum wind farm output coinciding with the minimum loading condition.

Fig. 6 shows voltage profiles on network buses for a selection of system types at various DG penetrations for the passive network voltage control scenario with and without SNOPs present at high DG penetration levels. SNOPs are observed to limit bus voltages throughout the networks even for large variations in the uncompensated case.

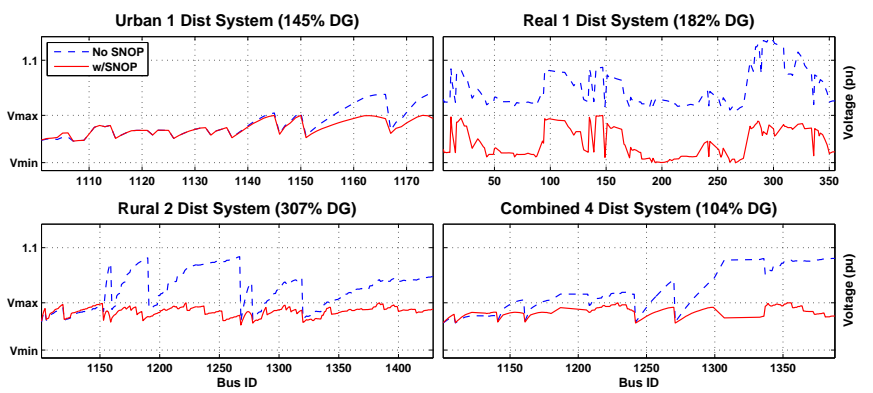

Figure 6. Voltage profiles at median and maximum DG penetration

\section{Economic Case Study}

Of particular interest for economic studies are the rural networks. Rural networks are prime candidates for highly concentrated installations of distributed/renewable generation such as a wind farms. This case study considers the Rural 2 data set with 28 MW DG capacity clustered near SNOP sites (placement scheme discussed in Section II-A). With two SNOPs, approximately $7 \mathrm{MW}$ of generation is installed near each SNOP end point. Note that the clustered placement strategy is considered for economic studies because large, fixed, DG installations are more likely to have a single owner and, hence, single earner of revenue with interest in what revenue the DG installation(s) can potentially generate, whereas the random placement paradigm assumes many generators with many owners.

As mentioned previously, the worst case for achieving maximum DG penetration is at minimum loading with maximum DG output. Maximum DG penetration will, however, vary according to loading conditions, generally increasing with increasing load. Fig. 7 illustrates this phenomena by considering load profile variations for a single day (lower line). The upper line in Fig. 7 indicates the maximum DG penetration coinciding with the loading conditions throughout the day. Maximum DG penetration tends to increase with increased loading due to a reduction in the voltage rise effect, i.e. greater portion of power generated by DGs is absorbed by local loads. Other voltage control strategies follow a similar pattern. Maximum generator output was correlated with yearly load profile data and integrated over the year to obtain a sum total of energy output per year.

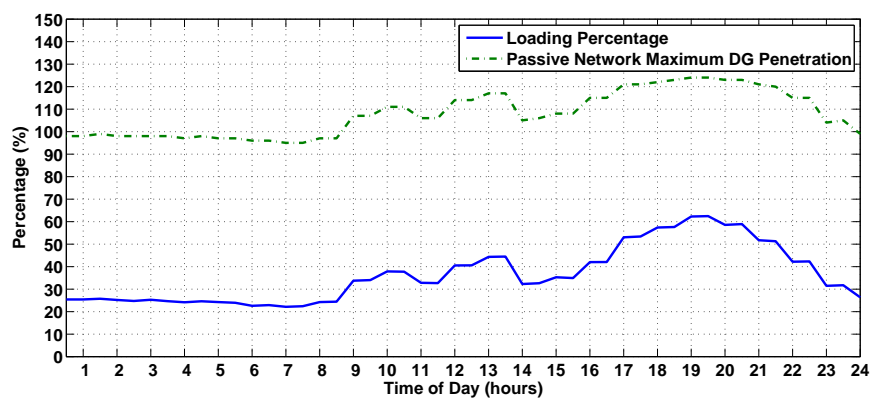

Figure 7. Maximum DG Penetration Percentage for given network loading of the Rural 2 system with the 'Passive Network' voltage control strategy

Since we are considering the same DG capacity installed 
for each voltage control option, maximum DG penetration limitations would require the owner of the DG installation to curtail generation so as not to adversely affect the network voltage. When comparing voltage control scenarios, the total generation (energy) curtailed per year can be considered revenue lost versus zero curtailment, i.e. continuous $28 \mathrm{MW}$ output throughout the year. Fig. 8 shows the resulting total generation per year, and lost revenue, for each voltage control option considered. The $28 \mathrm{MW}$ installation figure was chosen such that very little generation would be curtailed when utilizing the best performing voltage control strategy, which in both cases is the combined SNOP with Dynamic OLTC option. Similar results were obtained for the Rural 1 network. Note that a revenue of $£ 30 / \mathrm{MWh}$ is considered when quoting earnings; this should be considered an estimate of foregone revenue for an installation of this magnitude.

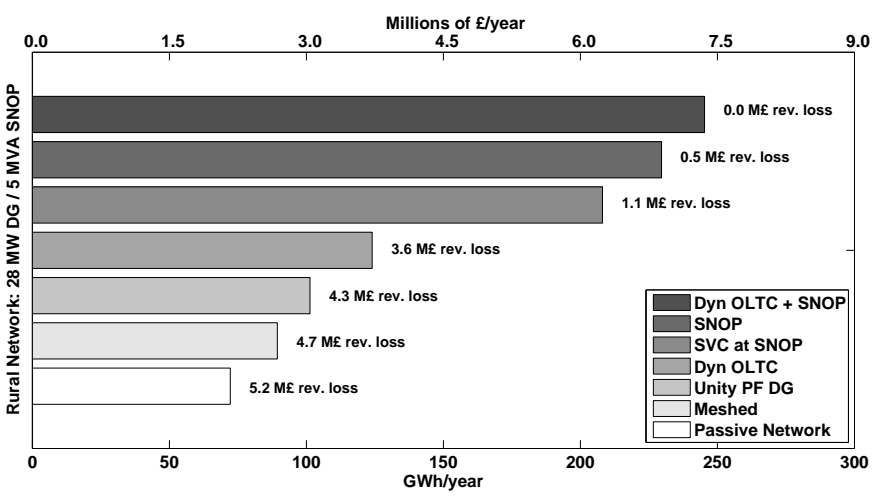

Figure 8. Total generation integrated over year and revenue gain/loss figures for $50 \mathrm{MW}$ installation with 5 MVA SNOPs installed

\section{CONCLUSIONS}

Based on the results presented in this paper, the presence of SNOPs clearly facilitates a large increase in possible DG penetration when compared with the uncompensated (passive network) case, and also when compared with other options for voltage control. While much of the benefit provided by the SNOP is due to its reactive power capabilities, as shown by comparing the reactive power compensation at SNOP site case versus the $S N O P$ case, it is clear that there is some added benefit by the addition of active power transfer capability (see Fig. 8). The additional benefits of SNOPs provided by active power transfer capability should be considered when evaluating a potential SNOP installation. The performance of the SNOP for this application is observed to increase with increased SNOP rating; as such, SNOPs could be rated to meet very high DG installation requirements if necessary.

By potentially increasing the revenue of DG developers by reducing levels of generation curtailment or allowing for increased capacity of installation, a sharing scheme can be established between DNOs and large DG developers for the costs associated with SNOP installation and maintenance. Furthermore, if incentives are put in place for the facilitation of DG, this could further add to the economic viability of SNOP equipment installation.

\section{REFERENCES}

[1] T. Ackermann and V. Knyazkin, "Interaction between distributed generation and the distribution network: operation aspects," in Transmission and Distribution Conference and Exhibition 2002: Asia Pacific. IEEE/PES, vol. 2, pp. 1357-1362 vol.2, 2002.

[2] F. Kupzog, H. Brunner, W. Pruggler, T. Pfajfar, and A. Lugmaier, "Dg demonet-concept - a new algorithm for active distribution grid operation facilitating high dg penetration," in Industrial Informatics, 2007 5th IEEE International Conference on, vol. 2, pp. 1197-1202, 2007.

[3] S. N. Liew and G. Strbac, "Maximising penetration of wind generation in existing distribution networks," IEE Proceedings - Generation, Transmission and Distribution, vol. 149, no. 3, pp. 256-262, 2002.

[4] C. M. Hird, H. Leite, N. Jenkins, and H. Li, "Network voltage controller for distributed generation," Generation, Transmission and Distribution, IEE Proceedings-, vol. 151, pp. 150-156, April 2004.

[5] G. Strbac, N. Jenkins, M. Hird, P. Djapic, and G. Nicholson, "Integration of operation of embedded generation and distribution networks," tech. rep., May 2002.

[6] J.-E. Kim, H. Kita, T. Tezuka, and Y. Nishikawa, "Methods of determining introduction limits of dispersed generation systems in a distribution system," Electrical Engineering in Japan, vol. 120, no. 4, pp. 48-58, 1997.

[7] G. Celli, F. Pilo, G. Pisano, V. Allegranza, R. Cicoria, and A. Iaria, "Meshed vs. radial mv distribution network in presence of large amount of dg," in Power Systems Conference and Exposition, 2004. IEEE PES, pp. 709-714 vol.2, 2004.

[8] United Kingdom Generic Distribution System project. University of Strathclyde, available: http://monaco.eee.strath.ac.uk/ukgds/.

[9] J. A. Momoh, Electric power system applications of optimization. Marcel Dekker, 2005.

[10] T. E. Kim and J. E. Kim, "A method for determining the introduction limit of distributed generation system in distribution system," in Power Engineering Society Summer Meeting, 2001. IEEE, vol. 1, pp. 456-461 vol.1, 2001.

[11] L. H. Willis, Power Distribution Planning Reference Book. CRC Press, 2004.

[12] J. Crabtree, Y. Dickson, L. Kerford, and A. Wright, "Methods to accommodate embedded generation without degrading network voltage regulation," tech. rep., 2001.

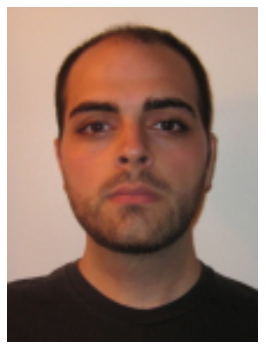

Jeffrey M. Bloemink (S'05) received his B.A.Sc. and M.A.Sc. degrees from the University of Toronto in 2005 and 2007, respectively. He is currently a $\mathrm{PhD}$ candidate attending Imperial College London, UK. His research interests include integration of power electronics in distribution systems as well as distributed generation coordination and control.

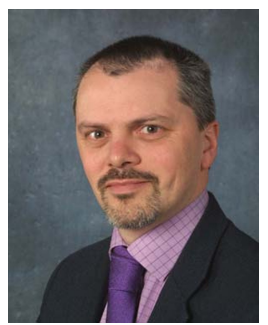

Timothy C. Green (M'1989, SM'02) received the B.Sc. degree (first class honours) in electrical engineering from Imperial College London, U.K., in 1986 and the Ph.D. degree in electrical engineering from Heriot-Watt University, Edinburgh, U.K., in 1990. He was a Lecturer at Heriot Watt University until 1994 and is now a Professor of electrical power Engineering at Imperial College London, deputy head of Control and Power Group and Deputy head of the Department of Electrical and Electronic Engineering. His research interests are power electronic and control to enhance power quality and power delivery. This covers interfaces and controllers for distributed generation, micro-grids, FACTS, autonomous active distribution networks, distribution network optimization. $\mathrm{He}$ has an additional line of research in power MEMS and energy scavenging. Professor Green is a Chartered Engineer in the U.K. and MIEE. 